\title{
The favorable role of homozygosity for killer immunoglobulin-like receptor (KIR) A haplotype in patients with advanced-stage classic Hodgkin lymphoma
}

Giorgio La Nasa ${ }^{1,2^{*}}$, Marianna Greco ${ }^{2}$, Roberto Littera ${ }^{3}$, Sara Oppi², Ivana Celeghini ${ }^{4}$, Rossella Caria ${ }^{2}$, Sara Lai ${ }^{3}$, Rita Porcella ${ }^{3}$, Massimo Martino ${ }^{5}$, Alessandra Romano ${ }^{6}$, Francesco Di Raimondo ${ }^{6}$, Andrea Gallamini ${ }^{7}$,

Carlo Carcassi $^{8}$ and Giovanni Caocci, ${ }^{1,2}$

\begin{abstract}
Background: Interim positron emission tomography after 2 cycles of ABVD (iPET-2) is a good predictor of outcome in advanced-stage classic Hodgkin lymphoma. So far, there are no other prognostic biomarkers capable of identifying chemotherapy refractory patients with comparable accuracy. Despite the considerable amount of evidence suggesting that antitumor immune surveillance is downregulated in classic Hodgkin lymphoma $(\mathrm{cHL})$, few data exist on the impairment of natural killer cell function and the role of their killer immunoglobulin-like receptors (KIRs).
\end{abstract}

Methods: We investigated KIR gene frequencies, KIR haplotypes, and KIR-ligand combinations in a cohort of 135 patients with advanced-stage classic Hodgkin lymphoma and 221 healthy controls. We furthermore evaluated the correlation of KIR genes and KIR haplotypes with the achievement of negative iPET-2.

Results: In the cohort of patients, the 5-year overall survival and progression-free survival were 93.6 and $79 \%$, respectively. Homozygosity for KIR A haplotype and the HLA-C1 KIR ligand (KIR-AA/C1C1) was significantly higher in healthy controls $(15.7$ vs. $4.8 \%, p=0.001)$. The KIR-AA genotype resulted to have a significant predictive power for achieving iPET-2 negativity $(p=0.039)$.

Conclusions: Homozygosity for KIR A haplotype offers protection against classic Hodgkin lymphoma. The association found for the KIR-AA genotype and achievement of negative iPET-2 suggests that KIR-AA could be used in clinical practice to enhance the chemosensitivity predictive power of iPET-2. Our results point to the possibility of adapting treatment strategies based on the combination of KIR biomarkers and PET scan.

Keywords: Hodgkin lymphoma, Killer immunoglobulin-like receptors, KIR A haplotype, Natural killer cells, Positron emission tomography

\footnotetext{
*Correspondence: lanasa@tiscali.it

${ }^{1}$ Hematology Unit, Department of Medical Sciences "M. Aresu", University of Cagliari, Via Is Guadazzonis, 3, 09126 Cagliari, Italy

${ }^{2}$ Bone Marrow Transplant Center, R. Binaghi Hospital, ASL 8, Cagliari, Italy

Full list of author information is available at the end of the article
} 


\section{Background}

Cure rates in classic Hodgkin lymphoma (cHL) range from 70 to $90 \%$, but complete recovery of patients is burdened by long-term toxicities of chemoradiation treatment, particularly heart and lung injury and secondary neoplasms [1]. Although in recent years interim $\left[{ }^{18} \mathrm{~F}\right]$-fluoro-2-deoxy-D-glucose positron emission tomography carried out after 2 cycles of doxorubicin, bleomycin, vinblastine, dacarbazine (ABVD) treatment (iPET-2) has emerged as a powerful predictor of treatment outcome in advanced-stage cHL patients [2], no other biomarkers have been shown capable of predicting treatment outcome at baseline with the same accuracy. Because of its unique histology, $\mathrm{cHL}$ is an interesting study model for the identification of new immunological and immunogenetic factors that may confer susceptibility to tumor or influence response to treatment [3]. In fact, the peculiar architecture of "malignant lymphogranuloma" is characterized by the presence of only a few neoplastic cells-Hodgkin-Reed-Sternberg (HRS) cells-growing in a microenvironment rich with immune system cells that are incapable of mounting an effective antitumor response [4].

Despite the considerable amount of evidence suggesting that antitumor immune surveillance is downregulated in cHL [5], few data exist on the impairment of natural killer (NK) cell function in cHL. NK cells are a key component of the innate immune system and play an important role in antitumor surveillance. Their activity is regulated by several receptor families, including killer cell immunoglobulin-like receptors (KIRs) which recognize human leukocyte antigen (HLA) class I molecules expressed on target cells [6]. NK cells express different combinations of the KIR2DL1, KIR2DL2, KIR2DL3, KIR2DL4, KIR2DL5, KIR3DL1, KIR3DL2, and KIR3DL3 inhibitory KIR genes and KIR2DS1, KIR2DS2, KIR2DS3, KIR2DS4, KIR2DS5, and KIR3DS1 activating KIR genes [7]. The KIR gene family has been divided into two broad groups of haplotypes according to gene content. KIR A haplotypes encode the KIR2DL3, KIR2DL1, KIR3DL1, and KIR3DL2 inhibitory genes and the KIR2DS4 activating gene. These haplotypes have identical KIR gene content, but can differ for allelic polymorphism. KIR B haplotypes contain various combinations of both activating and inhibitory KIR genes and show less allelic polymorphism $[8,9]$.

KIR receptors recognize four mutually exclusive epitopes carried by HLA-A, HLA-B, and HLA-C class I molecules: C1, C2, Bw4, and A3/A11. HLA-C is known to consist of two functional classes of allotypes, depending on an amino acid dimorphism at residue 80 of the $\alpha 1$ helix. The $\mathrm{C} 1$ epitope is carried by HLA-C allotypes with asparagine at position 80 and by some HLA-B alleles in the Asian population that have asparagine at position 80 and valine at position 76 . The $\mathrm{C} 2$ epitope is carried by HLA-C allotypes characterized by a lysine at position 80 of the $\alpha 1$-helix. The Bw4 epitope is carried by HLA-A and HLA-B allotypes that have arginine at position 83 . The A3/11 epitope of HLA-A is carried by two HLA-A allotypes, HLA-A*03 and HLA-A*11 $[10,11]$.

The diversity in gene content of KIR haplotypes and the extensive polymorphism of HLA and KIR genes make it possible for an individual to express KIR genes for which there are no HLA ligands and vice versa, which thus determines extremely variable immunogenetic profiles among individuals [12]. KIR A haplotypes seem to provide better immune surveillance against viral infections and tumor cells, whereas KIR B haplotypes seem to have a favorable role during pregnancy [10].

Although there is a growing interest in the role of NK cells in oncohematologic disorders [13-17], to the best of our knowledge, there is only one previous report investigating the possible involvement of KIR genes in cHL [18]. To gain further insight into the role of NK cells in cHL and their possible impact on response to treatment, we investigated KIR gene frequencies, KIR haplotypes, and KIR-ligand combinations in a cohort of advanced-stage cHL patients compared to healthy controls. Moreover, we evaluated the correlation of KIR genes and KIR haplotypes with the achievement of negative iPET-2 and their possible impact on progression-free survival (PFS).

\section{Methods}

\section{Ethics, consent, and permissions}

In accordance with the 1975 guidelines of the Declaration of Helsinki and after obtaining approval for the study from the four competent local Ethics Committees (Cagliari, Catania, Cuneo, and Reggio Calabria), the medical staff of each participating center exhaustively informed their patients of the potential risks and benefits of the planned treatment and the need for periodical clinical and laboratory checkups.

\section{Patients, healthy controls, and treatment protocols}

One hundred and thirty-five patients with advancedstage $\mathrm{cHL}$ were included in the study. All patients were enrolled in the international prospective multicenter clinical trial HD 0607 (NCT00795613). A complete data set containing all the following parameters was collected at baseline: age, sex, histological subtype, presence of Bsymptoms (fever, weight loss exceeding $10 \%$ of body weight in 6 months, night sweats), extranodal involvement or bulky mass, absolute lymphocyte and neutrophil count, hemoglobin and albumin, Ann Arbor stage, and International Prognostic Score (IPS) [19].

All the patients received two courses of standard ABVD treatment before undergoing iPET-2 to assess 
tumor chemosensitivity. Patients were stratified into two groups according to the results of iPET-2. Patients with positive iPET-2 were randomly assigned to receive four courses of escalated BEACOPP (rituximab, bleomycin, etoposide, doxorubicin, cyclophosphamide, vincristine, procarbazine, prednisone) or R-BEACOPP (BEACOPP supplemented with rituximab), followed by four courses of baseline BEACOPP or R-BEACOPP. Patients with a negative iPET-2 scan continued with four more courses of ABVD. Patients in complete remission with a negative final PET scan who presented bulky disease at baseline or showed a PET-negative residual mass at the end of chemotherapy were randomly assigned to receive consolidation radiotherapy or no further treatment.

A group of 221 healthy subjects (104 males and 117 females) with a mean age of 35 (range $25-55$ years) were selected for control purposes.

\section{HLA and KIR typing}

HLA typing was performed in healthy controls and patients using commercially available PCR kits with sequencespecific primers (HLA-A-B-C SSP Combi Tray, Olerup, Stockholm, Sweden, and Micro SSP ${ }^{\mathrm{rm}}$ Generic HLA class I typing tray SSP1L, One Lambda, Inc., Canoga Park, CA, USA). HLA-C groups $\mathrm{C} 1$ and $\mathrm{C} 2$ and HLA-Bw4 were determined by high-resolution typing for the alleles at the HLA-A, HLA-B, and HLA-C loci using a polymerase chain reaction-sequence-specific primer (PCR-SSP) method according to the manufacturers' instructions (allele-specific PCR-SSP kits: Olerup SSP AB, Stockholm, Sweden).

Distribution of KIR genes (KIR2DL1, KIR2DL2, KIR2DL3, KIR2DL4, KIR2DL5A, KIR2DL5B, KIR2DS1, KIR2DS2, KIR 2DS3, KIR2DS4, KIR2DS5, KIR3DL1, KIR3DL2, KIR3DL3, KIR3DS1) were determined by PCR-SSP using a previously reported method [20, 21].

Patients were stratified into two groups according to homozygosity for KIR A haplotype (KIR genotype AA) and heterozygosity or homozygosity for KIR B haplotype (KIR genotypes $\mathrm{AB}$ and $\mathrm{BB}$, referred together as KIR genotype Bx) [21, 22].

\section{Statistical analysis}

KIR gene frequencies, KIR haplotypes, and KIR-ligand combinations were compared between $\mathrm{cHL}$ patients and healthy controls as well as between cHL patients who achieved negative iPET-2 and those who did not. The abovementioned set of independent variables was included in univariate and multivariate analyses. Where appropriate, significant differences were calculated using Fisher's two-sided exact test or Pearson's chi-squared test. Variables with a $p$ value lower than 0.2 in univariate analysis were included in multivariate analysis using a multi-step forward binary logistic regression model, where negative iPET-2 scan was considered a dependent variable.
Only $p$ values $\leq 0.05$ were considered to be statistically significant. The probability of achieving overall survival (OS) and PFS was calculated using the Kaplan-Meier method. Comparisons were also made to assess PFS in patient subgroups according to KIR haplotype or iPET results. The log-rank test was used to compare the groups of patients.

\section{Results}

Characteristics of patients and controls

The demographic and clinical characteristics of 135 advanced-stage cHL patients (67 males and 68 females, mean follow-up 35 months, range 8-60) and 221 controls are shown in Table 1 . The overall mean age at diagnosis was 34 years (range 15-69). The large majority of patients (80\%) had a nodular sclerosing histological subtype. Bulky mass and "B" symptoms were found in 40.7 and $54.1 \%$, respectively. According to the HD 0607 inclusion criteria, all patients belonged to advanced Ann Arbor stages (IIb-IV) with an unfavorable IPS in $28.9 \%$ of cases. The demographic characteristics of the 221 healthy controls ( 104 males and 117 females) with a mean age of 35 (range 25-55 years) were not statistically different from the cHL cohort.

\section{KIR gene frequencies, KIR haplotypes, and KIR-HLA ligand combinations in $\mathrm{CHL}$ patients and controls}

Table 2 shows KIR gene frequencies, KIR haplotypes, and KIR-HLA ligand combinations in $135 \mathrm{cHL}$ patients and 221 healthy controls. No significant differences were found between patients and controls for the frequencies of the single activating and inhibitory KIR genes. Overall, the healthy controls had significantly higher frequencies of the HLA-C1 ligand $(\mathrm{C} 1 / \mathrm{C} 1$ or $\mathrm{C} 1 / \mathrm{C} 2)$ compared to patients (72.7 vs. $60 \%, p=0.014$ ); on the other hand, frequency of the HLA-C2 ligand $(\mathrm{C} 2 / \mathrm{C} 2$ or $\mathrm{C} 1 / \mathrm{C} 2)$ was significantly higher in cHL patients ( 88.9 vs. $76 \%, p=0.003$ ). No significant differences were found for frequencies of the HLA-Bw4 ligand within the two groups. Homozygosity for KIR A haplotype combined with homozygosity for the HLA-C1 ligand (KIRAA/C1C1) was significantly higher in healthy controls ( 15.7 vs. $4.8 \%, p=0.001$ ). The analysis of the combinations of KIR genes and their specific ligands showed a significantly higher frequency of KIR3DS1 and KIR3DL1 when combined with the absence of their specific ligand HLA-Bw4 in cHL patients (15.5 vs. $6.3 \%, p=$ 0.006 and 33.3 vs. $21.3 \%, p=0.013$, respectively).

\section{KIR gene frequencies, KIR haplotypes, and KIR-HLA ligand combinations in $\mathrm{CHL}$ patients with positive or negative iPET-2 results}

Table 3 shows HLA and KIR gene frequencies, KIR haplotypes, and the combinations between KIRs and their 
Table 1 Characteristics of 135 patients with advanced-stage classic Hodgkin lymphoma and 221 healthy controls

\begin{tabular}{|c|c|c|c|c|}
\hline \multirow{2}{*}{$\overline{\text { Sex, no. (\%) }}$} & \multicolumn{2}{|c|}{ Patients $n=135$} & \multirow[t]{2}{*}{ Controls $n=221$} & \multirow{2}{*}{$p$ value } \\
\hline & & & & \\
\hline Male & 67 & $(49.6)$ & $104(47.1)$ & NS \\
\hline Female & 68 & $(50.4)$ & $117(52.9)$ & NS \\
\hline Age at diagnosis, mean (range) & 34 & $(15-69)$ & $35(25-55)$ & NS \\
\hline \multicolumn{5}{|l|}{ Disease subtype, no. (\%) } \\
\hline Nodular sclerosing & 108 & $(80.0)$ & & \\
\hline Mixed-cellularity & 14 & $(10.4)$ & & \\
\hline Lymphocyte-rich & 9 & $(6.7)$ & & \\
\hline Lymphocyte-depleted & 4 & $(2.9)$ & & \\
\hline Albumin, g/dl, mean (range) & 3.8 & $(2.2-5.1)$ & & \\
\hline Hemoglobin, g/dl, mean (range) & 13.0 & $(8.3-14.2)$ & & \\
\hline Lymphocytes, $\times 10^{3} / \mu \mathrm{L}$, mean (range) & 1.75 & $(0.3-4.9)$ & & \\
\hline Neutrophils, $\times 10^{3} / \mu \mathrm{L}$, mean (range) & 8.55 & $(2.1-35.8)$ & & \\
\hline Bulky mass, no. (\%) & 55 & $(40.7)$ & & \\
\hline "B" symptoms, no. (\%) & 73 & $(54.1)$ & & \\
\hline Extranodal extension, no. (\%) & 42 & $(31.1)$ & & \\
\hline IPS score $\geq 4$ (unfavorable in advanced stages) & 39 & $(28.9)$ & & \\
\hline Ann Arbor stage 1-2a, no. (\%) & 0 & $(0)$ & & \\
\hline Stage $2 b$, no. (\%) & 57 & $(42.2)$ & & \\
\hline Stage 3, no. (\%) & 45 & $(33.3)$ & & \\
\hline Stage 4, no. (\%) & 33 & $(24.5)$ & & \\
\hline
\end{tabular}

NS not significant

ligands in 33 patients who showed positive iPET-2 in comparison with 102 patients with negative iPET-2.

A significantly higher frequency of the KIR2DS2 activating KIR gene was found in patients with positive iPET-2 (81.8 vs. $50 \%, p=0.001)$. Homozygosity for KIR A haplotype was present with a significantly higher frequency in patients with negative iPET-2 (36.3 vs. $15.2 \% ; p=0.03)$. In particular, only 5 of the 33 patients with positive iPET-2 had KIR genotype AA, whereas the remaining 28 (84.8 \%) had KIR genotype $\mathrm{Bx}$. Homozygosity for KIR A haplotype remained the only predictive factor for the achievement of negative iPET$2(p=0.039)$. The analysis of HLA-KIR combinations showed that $\mathrm{CHL}$ patients with a positive iPET-2 scan had higher frequencies of the activating KIR genes, KIR2DS1 and KIR2DS2, combined with the presence of their specific ligands $\mathrm{C} 2$ and $\mathrm{C} 1$, respectively (KIR2DS1/ HLA-C2: 66.7 vs. $35.3 \%, p=0.03$; KIR2DS2/HLA-C1: 63.3 vs. $20.6 \%, p=0.004)$. Higher frequencies were also observed in this group of patients for the inhibitory KIR genes, KIR2DL1 and KIR2DL2, combined with the presence of their specific ligands $\mathrm{C} 2$ and $\mathrm{C} 1$, respectively (KIR2DL1/HLA-C2: 81.8 vs. $57.8 \%$, $p=0.021$; KIR2DL2/ HLA-C1: 62.7 vs. $32.3 \%, p=0.018)$.
Overall survival and progression-free survival following treatment and according to KIR haplotype and iPET-2 In the cohort of 135 patients, 5-year OS and PFS was 93.6 and $79 \%$, respectively (Fig. 1). PFS was significantly higher in patients who achieved negative iPET compared to those who did not ( 89.8 vs. $55.8 \%, p=0.0001$ ); among patients with negative iPET, those homozygous for KIR A haplotype had higher PFS rates compared to those carrying Bx haplotypes, although the difference was not statistically significant (92.3 vs. $85.3 \%$ ) (Fig. 2).

\section{Discussion}

Survival for advanced-stage cHL patients has increased to $70-85 \%$ over the past years, but the optimal balance of the risks and benefits of treatment is still a matter of debate. At 15 years from the end of treatment, the risk of dying from lymphoma is less than the risk of dying from therapy-related complications [23]. The main dilemma remains whether frontline intensive chemotherapy should be given to all patients or be restricted to patients who are poor responders to less intensive treatment approaches. So far, iPET-2 scan represents the only strong predictor of response to treatment [2]. There are currently no other available prognostic biomarkers capable of distinguishing 
Table 2 KIR and HLA gene frequencies, KIR haplotypes, and KIR-HLA ligand combinations in 135 advanced-stage classic HL patients and 221 healthy controls

\begin{tabular}{|c|c|c|c|c|c|c|}
\hline & Patients (135) & Percent & Controls (221) & Percent & $p$ & OR $(95 \% \mathrm{Cl})$ \\
\hline \multicolumn{7}{|l|}{ Activating KIR } \\
\hline KIR2DS1 & 72 & 53.3 & 97 & 43.9. & & \\
\hline KIR2DS2 & 78 & 57.8 & 125 & 56.6 & & \\
\hline KIR2DS3 & 54 & 40.0 & 77 & 34.9 & & \\
\hline KIR2DS4 & 124 & 91.9 & 202 & 91.4 & & \\
\hline KIR2DS5 & 42 & 31.1 & 75 & 33.9 & & \\
\hline KIR3DS1 & 53 & 39.3 & 88 & 39.8 & & \\
\hline \multicolumn{7}{|l|}{ Inhibitory KIR } \\
\hline KIR2DL1 & 132 & 97.8 & 215 & 97.2 & & \\
\hline KIR2DL2 & 80 & 59.2 & 126 & 57.0 & & \\
\hline KIR2DL3 & 123 & 91.1 & 188 & 85.0 & & \\
\hline KIR2DL4 & 135 & 100 & 221 & 100 & & \\
\hline KIR2DL5 & 78 & 57.8 & 116 & 52.5 & & \\
\hline KIR3DL1 & 135 & 100 & 206 & 93.2 & & \\
\hline KIR3DL2 & 135 & 100 & 219 & 99.1 & & \\
\hline KIR3DL3 & 135 & 100 & 221 & 100 & & \\
\hline \multicolumn{7}{|l|}{ KIR genotypes } \\
\hline $\mathrm{AA}$ & 42 & 31.1 & 66 & 29.9 & & \\
\hline $\mathrm{Bx}$ & 93 & 68.8 & 155 & 70.1 & & \\
\hline $\mathrm{AA} / \mathrm{C} 1-\mathrm{C} 1$ & 2 & 4.8 & 35 & 15.7 & 0.001 & $0.08(0.013-0.35)$ \\
\hline \multicolumn{7}{|l|}{ KIR ligands } \\
\hline $\mathrm{C} 1$ pres & 81 & 60.0 & 161 & 72.7 & 0.014 & $0.56(0.35-0.90)$ \\
\hline C2 pres & 120 & 88.9 & 168 & 76 & 0.003 & $2.5(1.31-4.90)$ \\
\hline HLA-Bw4 pres & 90 & 66.9 & 175 & 79.2 & & \\
\hline \multicolumn{7}{|l|}{ Activating KIR and their ligands } \\
\hline KIR2DS1 pres/HLA-C2 pres & 56 & 41.5 & 78 & 35.3 & & \\
\hline KIR2DS1 pres/HLA-C2 abs & 16 & 11.9 & 19 & 11.6 & & \\
\hline KIR2DS2 pres/HLA-C1 pres & 46 & 34.1 & 80 & 36.2 & & \\
\hline KIR2DS2 pres/HLA-C1 abs & 32 & 23.7 & 45 & 20.4 & & \\
\hline KIR3DS1 pres/HLA-Bw4 pres & 32 & 23.7 & 74 & 33.5 & & \\
\hline KIR3DS1 pres/HLA-Bw4 abs & 21 & 15.5 & 14 & 6.3 & 0.006 & $2.7(1.27-5.9)$ \\
\hline \multicolumn{7}{|l|}{ Inhibitory KIR and their ligands } \\
\hline KIR2DL1 pres/HLA-C2 pres & 100 & 74.1 & 164 & 74.2 & & \\
\hline KIR2DL1 pres/HLA-C2 abs & 32 & 23.7 & 51 & 23.1 & & \\
\hline KIR2DL2 pres/HLA-C1 pres & 47 & 34.8 & 83 & 37.6 & & \\
\hline KIR2DL2 pres/HLA-C1 abs & 32 & 23.7 & 43 & 19.5 & & \\
\hline KIR2DL3 pres/HLA-C1 pres & 78 & 57.8 & 132 & 59.7 & & \\
\hline KIR2DL3 pres/HLA-C1 abs & 45 & 33.3 & 56 & 25.3 & & \\
\hline KIR3DL1 pres/HLA-Bw4 pres & 90 & 63.7 & 159 & 71.9 & & \\
\hline KIR3DL1 pres/HLA-Bw4 abs & 45 & 33.3 & 47 & 21.3 & 0.013 & $1.9(1.1-3.1)$ \\
\hline
\end{tabular}

abs absent, pres present, OR odds ratio, $C l$ confidence interval, Ile isoleucine, Thr threonine 
Table 3 KIR and HLA gene frequencies, KIR haplotypes, and KIR-HLA ligand combinations in 135 advanced-stage classic Hodgkin lymphoma patients according to positive or negative interim PET-2 results

\begin{tabular}{|c|c|c|c|c|c|c|}
\hline & PET-2 positive no. 33 & $\% 24.4$ & PET-2 negative no. 102 & $\% 75.6$ & $p$ & OR $(95 \% \mathrm{Cl})$ \\
\hline \multicolumn{7}{|l|}{ Activating KIR } \\
\hline KIR2DS1 & 23 & 69.7 & 49 & 48.0 & & \\
\hline KIR2DS2 & 27 & 81.8 & 51 & 50.0 & 0.001 & $4.5(1.6-13.3)$ \\
\hline KIR2DS3 & 16 & 48.5 & 38 & 37.3 & & \\
\hline KIR2DS4 & 28 & 84.9 & 96 & 94.1 & & \\
\hline KIR2DS5 & 9 & 27.3 & 33 & 32.3 & & \\
\hline KIR3DS1 & 16 & 48.5 & 37 & 36.3 & & \\
\hline \multicolumn{7}{|l|}{ Inhibitory KIR } \\
\hline KIR2DL1 & 33 & 100 & 99 & 97.1 & & \\
\hline KIR2DL2 & 22 & 66.7 & 58 & 56.9 & & \\
\hline KIR2DL3 & 30 & 90.9 & 93 & 91.2 & & \\
\hline KIR2DL4 & 33 & 100 & 102 & 100 & & \\
\hline KIR2DL5 & 20 & 60.6 & 58 & 56.9 & & \\
\hline KIR3DL1 & 33 & 100 & 102 & 100 & & \\
\hline KIR3DL2 & 33 & 100 & 102 & 100 & & \\
\hline KIR3DL3 & 33 & 100 & 102 & 100 & & \\
\hline \multicolumn{7}{|l|}{ KIR genotypes } \\
\hline AA & 5 & 15.2 & 37 & 36.3 & 0.03 & $0.31(0.1-0.95)$ \\
\hline $\mathrm{Bx}$ & 28 & 84.8 & 65 & 63.7 & & \\
\hline \multicolumn{7}{|l|}{ KIR ligands } \\
\hline C1 pres & 22 & 66.7 & 74 & 72.5 & & \\
\hline C2 pres & 27 & 81.8 & 88 & 86.3 & & \\
\hline HLA-Bw4 pres & 24 & 72.7 & 66 & 64.7 & & \\
\hline \multicolumn{7}{|l|}{ Activating KIR and their ligands } \\
\hline KIR2DS1 pres/HLA-C2 pres & 22 & 66.7 & 36 & 35.3 & 0.03 & $7.94(0.496-173)$ \\
\hline KIR2DS1 pres/HLA-C2 abs & 1 & 3.3 & 13 & 12.7 & & \\
\hline KIR2DS2 pres/HLA-C1 pres & 21 & 63.3 & 21 & 20.6 & 0.004 & $5.0(1.55-16.8)$ \\
\hline KIR2DS2 pres/HLA-C1 abs & 6 & 18.2 & 30 & 29.4 & & \\
\hline KIR3DS1 pres/HLA-Bw4 pres & 11 & 33.3 & 17 & 16.7 & & \\
\hline KIR3DS1 pres/HLA-Bw4 abs & 5 & 15.6 & 20 & 19.6 & & \\
\hline \multicolumn{7}{|l|}{ Inhibitory KIR and their ligands } \\
\hline KIR2DL1 pres/HLA-C2 pres & 27 & 81.8 & 59 & 57.8 & 0.021 & $3.05(1.07-9.11)$ \\
\hline KIR2DL1 pres/HLA-C2 abs & 6 & 18.2 & 40 & 42.2 & & \\
\hline KIR2DL2 pres/HLA-C1 pres & 19 & 62.7 & 33 & 32.3 & 0.018 & $4.8(1.2-23.1)$ \\
\hline KIR2DL2 pres/HLA-C1 abs & 3 & 9.1 & 25 & 24.5 & & \\
\hline KIR2DL3 pres/HLA-C1 pres & 23 & 69.7 & 55 & 53.9 & & \\
\hline KIR2DL3 pres/HLA-C1 abs & 7 & 21.2 & 38 & 37.3 & & \\
\hline KIR3DL1 pres/HLA-Bw4 pres & 22 & 66.7 & 65 & 63.7 & & \\
\hline KIR3DL1 pres/HLA-Bw4 abs & 11 & 33.3 & 37 & 36.3 & & \\
\hline
\end{tabular}

abs absent, pres present, $O R$ odds ratio, $\mathrm{Cl}$ confidence interval, lle isoleucine, Thr threonine

patients requiring more or less intensive treatment. For this reason, many ongoing studies focus their efforts on the definition of new predictive factors for prognosis and response to treatment in cHL. Ideally, risk assessment should combine innovative predictive biomarkers with new imaging technologies [24-27]. 


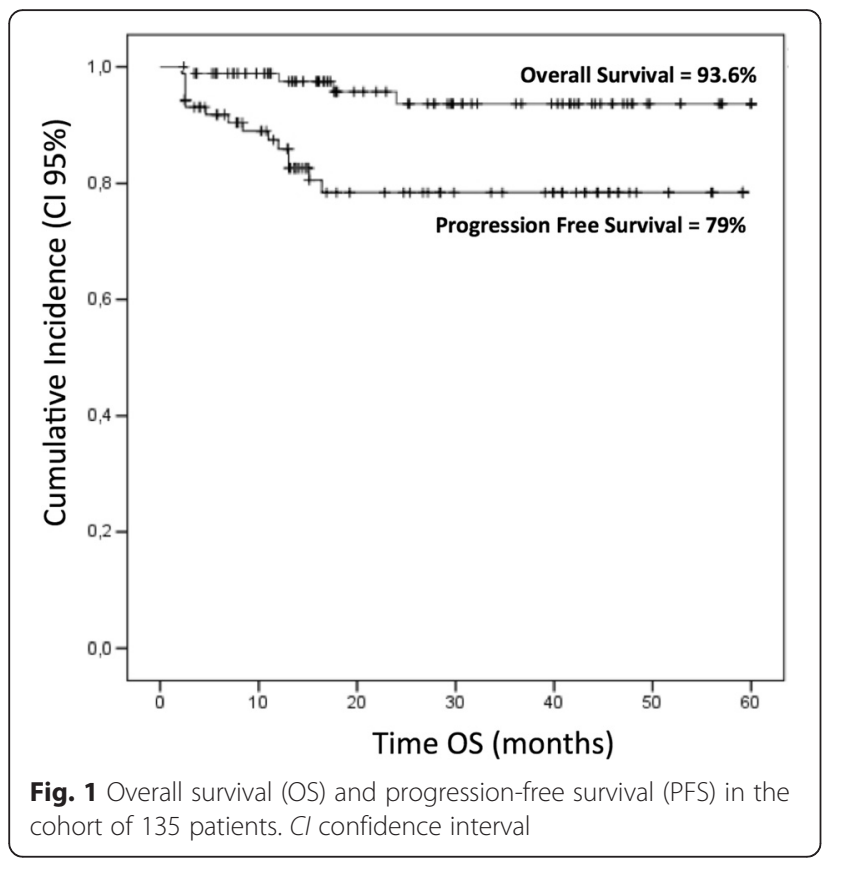

Fig. 1 Overall survival (OS) and progression-free survival (PFS) in the cohort of 135 patients. Cl confidence interval
Classic HL represents an extremely interesting study model for the assessment of immunologic and immunogenetic factors that may confer susceptibility to tumors or facilitate tumor immune escape mechanisms $[4,5]$. Currently few data exist on the role of impaired NK cell function in immune escape of HRS cells.

Besson et al. investigated association between KIRs and $\mathrm{cHL}$ in a family study [18]. They provide evidence that activating KIR genes, in particular KIR2DS1 and KIR3DS1, may exert protection in cHL. In our cohort of patients, we found a significantly higher frequency for KIR3DS1 in the absence of its specific Bw4 ligand, suggesting that this activating KIR gene cannot properly exert its protective function. No other significant differences were observed between cHL patients and controls for single KIR gene frequencies. Vice versa, when we analyzed $\mathrm{C} 1$ and $\mathrm{C} 2$ epitopes, we found that $\mathrm{C} 2$ had a significantly higher frequency in cHL patients ( 88.9 vs. $76 \%, p=0.008$ ). Conversely, the $\mathrm{C} 1$ epitope was significantly higher in healthy controls (72.7 vs. $60, p=0.036$ ) (Table 2). Interestingly, homozygosity for KIR A haplotype and HLA-C1 ligand (KIRAA/C1C1) was significantly higher in healthy controls, suggesting a possible protective role in $\mathrm{cHL}$ (15.7 vs. $4.8 \%, p=0.001$ ) (Table 2 ).

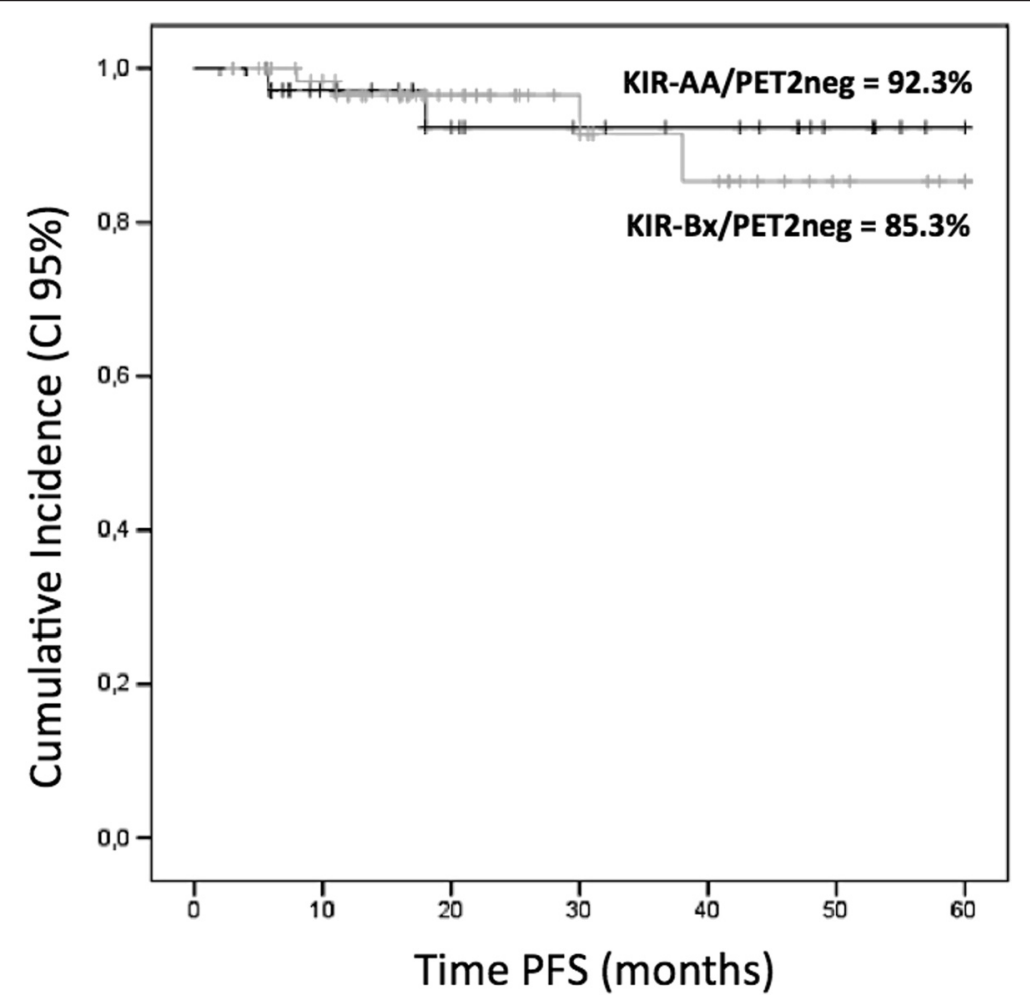

Fig. 2 Progression-free survival (PFS) in the cohort of 102 patients with negative interim PET, stratified in two groups according to KIR haplotype $(p=\mathrm{NS})$. Cl confidence interval 
In a recent review, Parham and Moffett suggest that homozygosity for HLA-C1 and KIR haplotype A could be associated to a better immune response with resistance to viral infection [10]. It is possible that the fixed gene content of KIR A haplotypes with their highly polymorphic receptors provides better immune surveillance against viral infections, whereas KIR B haplotypes with their greater variation in gene content and moderate allelic polymorphism could have an important role in immune-tolerance during pregnancy [10]. Our data seem to suggest a protective role for the KIRAA/C1C1 genotype in CHL.

In our study, KIR A haplotype was the only factor significantly associated to a negative iPET-2 result suggesting its favorable role in the achievement of better response to standard chemotherapy. Moreover, the combination of negative iPET-2 with homozygosity for KIR A haplotype seems to represent a favorable combination for identifying patients responsive to standard courses of chemotherapy (PFS $=92.3 \%$ ) (Fig. 2).

Finally, we found that cHL patients with iPET-2 positivity had higher frequencies of the KIR-ligand combinations KIR2DS1/HLA-C2 and KIR3DS2/HLA-C1. The interaction between KIR2DS1, KIR2DS2, and their HLA-C ligands might increase the production of tumor growth factor (TGF)- $\beta$ by NK cells. It has been suggested that TGF- $\beta$ may reduce tumor lysis mediated by NK cells and stimulate the production of proinflammatory cytokines such as tumor necrosis factor alpha $(\mathrm{TNF}-\alpha)$ and interferon gamma (IFN- $\gamma)$ [28].

\section{Conclusions}

Our results, though preliminary, seem to show that homozygosity for KIR A haplotype, alone or in combination with homozygosity for the HLA-C1 KIR ligand, confers protection to $\mathrm{cHL}$. The association found for the KIR-AA genotype and achievement of negative iPET-2 suggests that testing for KIR haplotypes could be used in clinical practice to enhance the power of iPET-2 in predicting better treatment outcome in cHL patients. Overall, our findings may represent a rationale for developing new risk-adapted treatment strategies based on the combination of KIR biomarkers and PET scans. They furthermore strengthen the hypothesis of innovative and targeted therapies aimed at modulating NK cell function. The use of anti-inhibitory-KIR antibodies could prove to be an effective way of blocking the binding interface of inhibitory KIRs with HLA class I ligands expressed by lymphoma cells, thus preventing a tolerogenic interaction and augmenting NK cell cytotoxicity [28-31].

\section{Competing interests}

The authors declare that they have no competing interests.

\section{Authors' contributions}

GLN, GC, and AG contributed to the conception and design. GC, MG, RL, SO, $I C, R C, S L, N O, M M, A R, F D R, C C, A G$, and GLN contributed to the collection and assembly of data. GC, MG, and RL contributed to the statistical analysis. GC and GLN contributed to the manuscript writing. GC, MG, RL, SO, IC, RC, $S L, N O, M M, A R, F D R, C C, A G$, and GLN contributed to the final approval of the manuscript.

\section{Acknowledgements}

We wish to thank Gruppo Italiano Terapie Innovative Linfomi (GITIL) for supporting this study. We are deeply grateful to all the patients who participated in this study. We are also grateful to Anna Maria Koopmans for professional writing assistance.

\section{Funding}

None

\section{Author details}

${ }^{1}$ Hematology Unit, Department of Medical Sciences "M. Aresu", University of Cagliari, Via Is Guadazzonis, 3, 09126 Cagliari, Italy. ${ }^{2}$ Bone Marrow Transplant Center, R. Binaghi Hospital, ASL 8, Cagliari, Italy. ${ }^{3}$ Regional Transplant Center, R. Binaghi Hospital, ASL 8, Cagliari, Italy. ${ }^{4}$ Hematology Department and BMT Unit, Azienda Ospedaliera S. Croce e Carle, Cuneo, Italy. ${ }^{5} \mathrm{Hematology}$ and Bone Marrow Transplant Unit, Azienda Ospedaliera BMM, Reggio Calabria, Italy. ${ }^{6}$ Division of Hematology, Azienda Policlinico-OVE, University of Catania, Catania, Italy. ${ }^{7}$ Research, Innovation and Statistics Department, Centre Antoine Lacassagne, Nice Cedex 2, France. ${ }^{8}$ Medical Genetics, Department of Medical Sciences "M. Aresu", University of Cagliari, Cagliari, Italy.

Received: 11 February 2016 Accepted: 5 March 2016

Published online: 16 March 2016

\section{References}

1. Diefenbach C, Advani R. Customized targeted therapy in Hodgkin lymphoma: hype or hope? Hematol Oncol Clin North Am. 2014;28:105-22.

2. Gallamini A, Barrington SF, Biggi A, Chauvie S, Kostakoglu L, Gregianin M, et al. The predictive role of interim positron emission tomography on Hodgkin lymphoma treatment outcome is confirmed using the 5-point scale interpretation criteria. Haematologica. 2014;99:1107-13.

3. Scott DW, Steidl C. The classical Hodgkin lymphoma tumor microenvironment: macrophages and gene expression-based modeling ASH Education Book. 2014;1:144-50.

4. Romano A, Vetro C, Caocci G, Greco M, Parrinello NL, Di Raimondo F, et al. Immunological deregulation in classic Hodgkin lymphoma. Mediterr J Hematol Infect Dis 2014; e2014039. doi:10.4084/MJHID.2014.039.

5. Visser L, Van den Berg A, Poppema S, Diepstra A. Microenvironment, crosstalk, and immune escape mechanisms. In: Engert A, Horning SJ, editors. Hodgkin lymphoma: a comprehensive update on diagnostics and clinics. Berlin: Springer Verlag; 2011. p. 49-58.

6. Purdy AK, Campbell KS. Natural killer cells and cancer: regulation by the killer cell Ig-like receptors (KIR). Cancer Biol The. 2009;8:2211-20.

7. Bashirova AA, Martin MP, McVicar DW, Carrington M. The killer immunoglobulin-like receptor gene cluster: tuning the genome for defense. Annu Rev Genomics Hum Genet. 2006;7:277-300.

8. Hsu KC, Chida S, Geraghty DE, Dupont B. The killer cell immunoglobulin-like receptor (KIR) genomic region: gene-order, haplotypes and allelic polymorphism. Immunol Rev. 2002;190:40-52.

9. Bontadini A, Testi M, Cuccia MC, Martinetti M, Carcassi C, Chiesa A, et al. Distribution of killer cell immunoglobulin-like receptors genes in the Italian Caucasian population. J Transl Med. 2006;4:44.

10. Parham P, Moffett A. Variable NK cell receptors and their MHC class I ligands in immunity, reproduction and human evolution. Nat Rev Immunol. 2013; 13:133-44.

11. O'Connor GM, Guinan KJ, Cunningham RT, Middleton D, Parham P, Gardiner CM. Functional polymorphism of the KIR3DL1/S1 receptor on human NK cells. J Immunol. 2007;178:235-41.

12. Parham P. MHC class I molecules and KIRs in human history, health and survival. Nat Rev Immunol. 2005;5:201-14.

13. Marin D, Gabriel IH, Ahmad S, Foroni L, De Lavallade H, Clark R, et al. KIR2DS1 genotype predicts for complete cytogenetic response and survival 
in newly diagnosed chronic myeloid leukemia patients treated with imatinib. Leukemia. 2012;26:296-302.

14. Bernal M, Garrido P, Jiménez P, Carretero R, Almagro M, López P, et al. Changes in activatory and inhibitory natural killer (NK) receptors may induce progression to multiple myeloma: implications for tumor evasion of $\mathrm{T}$ and NK cells. Hum Immunol. 2009;70:854-7.

15. Karabon L, Jedynak A, Giebel S, Wołowiec D, Kielbinski M, Woszczyk D, et al. $\mathrm{KIR} / \mathrm{HLA}$ gene combinations influence susceptibility to B-cell chronic lymphocytic leukemia and the clinical course of disease. Tissue Antigens. 2011;78:129-38.

16. Almalte Z, Samarani S, lannello A, Debbeche O, Duval M, Infante-Rivard C, et al. Novel associations between activating killer-cell immunoglobulin-like receptor genes and childhood leukemia. Blood. 2011;118:1323-8.

17. La Nasa G, Caocci G, Littera R, Atzeni S, Vacca A, Mulas O, et al. Homozygosity for killer immunoglobin-like receptor haplotype A predicts complete molecular response to treatment with tyrosine kinase inhibitors in chronic myeloid leukemia patients. Exp Hematol. 2013;41:424-31.

18. Besson C, Roetynck S, Williams F, Orsi L, Amiel C, Lependeven C, et al. Association of killer cell immunoglobulin-like receptor genes with Hodgkin's lymphoma in a familial study. PLoS One. 2007;2, e406.

19. Hasenclever D, Diehl V. A prognostic score for advanced Hodgkin's disease: international prognostic factors project on advanced Hodgkin's disease. N Engl J Med. 1998;339:1506-14.

20. Gagne K, Brizard G, Gueglio B, Milpied N, Herry P, Bonneville F, et al. Relevance of KIR gene polymorphisms in bone marrow transplantation outcome. Human Immunol. 2002:63:271-80.

21. Yawata M, Yawata N, McQueen KL, Cheng NW, Guethlein LA, Rajalingam R, et al. Predominance of group A KIR haplotypes in Japanese associated with diverse NK cell repertoires of KIR expression. Immunogenetics. 2002;54:543-50.

22. Middleton D, Gonzelez F. The extensive polymorphism of KIR genes. Immunology. 2010;129:8-19.

23. Hay AE, Meyer RM. Balancing risks and benefits of therapy for patients with favorable-risk limited-stage Hodgkin lymphoma: the role of doxorubicin, bleomycin, vinblastine, and dacarbazine chemotherapy alone. Hematol Oncol Clin North Am. 2014;28:49-63.

24. Tan KL, Scott DW, Hong F, Kahl BS, Fisher RI, Bartlett NL, et al. Tumor-associated macrophages predict inferior outcomes in classic Hodgkin lymphoma: a correlative study from the E2496 Intergroup trial. Blood. 2012;120:3280-7.

25. Scott DW, Chan FC, Hong F, Rogic S, Tan KL, Meissner B, et al. Gene expression-based model using formalin-fixed paraffin-embedded biopsies predicts overall survival in advanced-stage classical Hodgkin lymphoma. J Clin Oncol. 2013;31:692-700.

26. Meyer RM. EBV DNA: a Hodgkin lymphoma biomarker? Blood. 2013;121: $3541-2$.

27. Knecht H, Kongruttanachok N, Sawan B, Brossard J, Prévost S, Turcotte E, et al. Three dimensional telomere signatures of Hodgkin- and ReedSternberg cells at diagnosis identify patients with poor response to conventional chemotherapy. Transl Oncol. 2012:5:269-77.

28. Benson Jr DM, Bakan CE, Zhang S, Collins SM, Liang J, Srivastava S, et al. IPH2101, a novel anti-inhibitory KIR antibody, and lenalidomide combine to enhance the natural killer cell versus multiple myeloma effect. Blood. 2011; 118:6387-91.

29. Kohrt HE, Thielens A, Marabelle A, Sagiv-Barfi I, Sola C, Chanuc F, et al. Anti-KIR antibody enhancement of anti-lymphoma activity of natural killer cells as monotherapy and in combination with anti-CD20 antibodies. Blood. 2014;123:678-86.

30. Benson Jr DM, Hofmeister CC, Padmanabhan S, Suvannasankha A Jagannath S, Abonour R, et al. A phase 1 trial of the anti-KIR antibody IPH2101 in patients with relapsed/refractory multiple myeloma. Blood. 2012; 120:4324-33.

31. Murphy WJ, Parham P, Miller JS. NK cells, from bench to clinic. Biol Blood Marrow Transplant. 2012;18 Suppl 1:S2-7. doi:10.1016/j.bbmt.2011.10.033.

\section{Submit your next manuscript to BioMed Central and we will help you at every step:}

- We accept pre-submission inquiries

- Our selector tool helps you to find the most relevant journal

- We provide round the clock customer support

- Convenient online submission

- Thorough peer review

- Inclusion in PubMed and all major indexing services

- Maximum visibility for your research

Submit your manuscript at www.biomedcentral.com/submit
Biomed Central 